\title{
Caracterización de las actividades de tiempo libre de los adolescentes del municipio del Valle de San José-Santander y las expectativas frente a los programas de actividad física
}

\section{Characterization of the leisure activities of adolescents in the municipality of Valle de San José- Santander and expectations regarding physical activity programs}

\author{
Edwar Fabian Estévez-Gómez (iD; Luz Amelia Hoyos-Cuartas² (iD) \\ Universidad Pedagógica y Tecnológica de Colombia. Tunja - Colombia. edwar_fabian@hotmail.com \\ ²Universidad Pedagógica Nacional. Bogotá - Colombia.luzahoyos@yahoo.com
}

Cómo citar: Estévez-Gómez, E.D.; Hoyos-Cuartas, L.A. 2021. Caracterización de las actividades de tiempo libre de los adolescentes del municipio del Valle de San José-Santandery las expectativas frente a los programas de actividad física. Rev. Digit. Act. Fis. Deport. 7(1):e1793. http://doi.org/10.31910/rdafd.v7.n1.2021.1793

Artículo de acceso abierto publicado por Revista Digital: Actividad Física y Deporte, bajo una licencia Creative Commons CC BY-NC 4.0

Publicación oficial de la Universidad de Ciencias Aplicadas y Ambientales U.D.C.A, Institución de Educación Superior Acreditada de Alta Calidad por el Ministerio de Educación Nacional.

Recibido: Junio 9 de 2020 Aceptado: Octubre 28 de 2020 Editado por: Álvaro José Gracia Díaz

\section{RESUMEN}

Introducción: El buen uso del tiempo libre y la actividad física ayudan al mejoramiento de la calidad de vida y al desarrollo mental y físico saludable, particularmente, en la adolescencia. Por lo tanto, las comunidades escolares deben conocer las característicasy las expectativas que tiene susjóvenes. Objetivo: Caracterizar las actividades de tiempo libre de los adolescentes e identificar las expectativas que tienen frente a los programas de Actividad Física que ofrece el municipio del Valle de San José. Población y métodos: Con enfoque cuantitativo y diseño descriptivo-correlacional, se valoró una población total de 298 estudiantes de 14-19 años, de los colegios públicos y privados del municipio. Se utilizó un cuestionario ad hoc de preguntas cerradas y respuestas múltiples, que se analizó con el software IBM-SPSS Statistic versión 25. Resultados: Se encontró que, en estos adolescentes, con edad promedio de 15,2 $\pm 1,17$ años, las actividades que en su tiempo libre sobresalen son ver TV, escuchar música y estar en casa. El 72,1\% de los adolescentes realizan actividades físicas; sin embargo, solo $37,4 \%$ le dedica mínimo 60 minutos/día y 41,3\% incorporan prácticas deportivas o actividad física 3 veces/ semana. Respecto a la percepción de los programas de actividad física, el $66,1 \%$ reportan que no se han enterado y de existir, el 84,9\% irían hasta tres veces/ semana. En el análisis correlacional, se encontró relación significativa inversa $(p=0,009)$ entre edad y minutos/día que dedican a prácticas deportivas y actividad física, de manera que los adolescentes menores dedican más tiempo diario al deporte y los 
mayores tienen menor dedicación. Conclusión: Un alto porcentaje de adolescentes realizan actividad física, pero no con la frecuencia y duración óptima para su edad, aunque hay amplia motivación de participar en programas de actividad física; sería necesario ofertar programas que incrementen el interés de los adolescentes hacia una práctica más frecuente de actividad física, para un estilo de vida saludable.

Palabras clave: Actividad física; Tiempo libre; Adolescente; Ocio; Deporte.

\section{ABSTRACT}

Introduction: The good use of free time and physical activity help to improve the quality of life and healthy mental and physical development, particularly in adolescence. Therefore, school communities must know the characteristics and expectations of their youth. Objective: Characterize the leisure activities of adolescents and identify the expectations they have regarding the Physical Activity programs offered by the municipality of Valle de San José. Population and methods: With a quantitative approach and descriptive-correlational design, 298 students aged 14-19 years, from public and private schools in the municipality, were evaluated. An ad hoc questionnaire with closed questions and multiple answers was used, which was analyzed with the software IBM-SPSS Statistic version 25. Results: It was found that in these adolescents, with an average age of $15.2 \pm 1.17$ years, the activities that predominate in their free time are watching TV, listening to music and being at home. $72.1 \%$ of adolescents carry out physical activities. However, only $37.4 \%$ dedicate a minimum of 60 minutes / day and $41.3 \%$ incorporate sports or physical activity 3 times / week. Regarding the perception of physical activity programs, $66.1 \%$ report that they have not found out and if there are $84.9 \%$, they would go up to three times / week. In the correlational analysis, a significant inverse relationship ( $p=0.009$ ) was found between age and minutes / day devoted to sports and physical activity, so that younger adolescents spend more time daily in sports and older adolescents have less dedication. Conclusion: A high percentage of adolescents do physical activity but not with the frequency and optimal duration for their age, although there is ample motivation to participate in physical activity programs, it would be necessary to offer programs that increase the interest of adolescents towards a more frequent practice of activity Physics for a healthy lifestyle.

Keywords: Physical Activity; Free time; Adolescents; Leisure; Sport.

\section{INTRODUCCIÓN}

La adolescencia, se presenta como una etapa clave en la adquisición y consolidación de hábitos de práctica de actividad física o, incluso, del abandono de la misma (Baena \& Granero, 2015). Los bajos niveles de condición física de los adolescentes y los altos niveles de sobrepeso y obesidad de los estudiantes en edad escolar, asociado con niveles bajos de actividad física, entre otros factores, en la actualidad, generan una gran preocupación social. Los hábitos de vida de los adolescentes son cada vez más sedentarios y dedican la mayor parte de su tiempo libre a realizar actividades de tipo pasivo (Valera et al. 2011).

Siendo el tiempo libre, en algunas circunstancias, usado equivocadamente por los adolescentes, en actividades, como ver Tv, jugar video juegos y navegar por internet (Huertas et al. 2008).

Al empezar la adolescencia, los jóvenes entrarán a una etapa de cambios, tanto físicos como psicológicos, donde deben cumplir una serie de tareas sociales, escolares y personales; es ahí, donde una parte de esta población abandona la práctica de AF por motivos de tiempo, falta de motivaciones e interés. Es en esta etapa, donde se hace realmente necesario poner en marcha programas, estrategias en el tiempo libre, basados en los gustos de estos adolescentes, con fin que captar su interés y constancia en estas propuestas en su tiempo libre (Galvin, 2019).

La importancia de la práctica de actividad física en los adolescentes, se encuentra sustentada en el llamado de atención que hace la Organización Mundial de la Salud (OMS), con la alarmante cifra que afirma que la inactividad física es el cuarto factor de riesgo en lo que respecta a la mortalidad, a nivel mundial. Además de las recomendaciones que plantea para que los niños y jóvenes inviertan, como mínimo, 60 minutos diarios en actividades físicas de intensidad moderada a vigorosa (Organización Mundial de la Salud, 2020).

En la presente investigación, se desarrolló el proceso investigativo sobre las características de una 
población específica, como son los adolescentes en cuanto a sus datos sociodemográficos y las actividades que estos usualmente realizan en su tiempo libre; además, se indagó las expectativas que tienen frente a los diferentes programas existentes, manifestando cuáles son los programas y espacios que les gustaría que existieran, basados en sus gustos y necesidades reales.

\section{MATERIALES Y MÉTODOS}

Esta investigación tiene un enfoque cuantitativo, debido a que se centra en datos que son medibles y se deben analizar con métodos de estadística descriptiva y correlacional. El alcance del estudio es descriptivo, por cuanto se ocupa de caracterizar las variables de análisis: actividades de tiempo libre y expectativas frente a los programas de actividad física ofertados. Esta investigación, se desarrolló como un estudio no experimental, en el que se indagan diversos aspectos, que permiten recoger información, de manera independiente o conjunta, sobre las variables que se desea caracterizar en este estudio.

Participantes: La investigación, se desarrolló con la población total de adolescente escolares del municipio de Valle de San José-Santander, con edades entre 14 y 19 años. Los criterios de exclusión presentados son los adolescentes que están en el rango de edad, pero estudian en establecimiento educativos fuera del municipio y estudiantes en situación de discapacidad cognitiva, que les impide contestar la encuesta. Todos los participantes accedieron a participar de forma voluntaria y firmaron un asentimiento y un consentimiento informado. Instrumento: Se utilizó para la recolección de información, la encuesta para la caracterizar la actividad física en el tiempo libre en adolescentes y sus expectativas frente a los programas de actividades física y deportivas (ECAFTLA ad hoc). Es importante precisar, que si bien existen cuestionarios validados, enfocados en caracterizar las actividades de tiempo libre en adolescentes, no existe un cuestionario que, adicionalmente, indague sobre las expectativas que tienen dichos adolescentes, respecto a la formulación de programas de actividades físicas y deportivas, ofertados por la entidad municipal responsable. Por este motivo fue necesario, para la presente investigación, diseñar un cuestionario ad hoc.
El cuestionario está compuesto por 17 ítems, con preguntascerradas yabiertas, de respuestas múltiples, que exploran las características sociodemográficas y las variables de la investigación. Este cuestionario fue elaborado sobre la base de los aportes de los diversos autores, quienes utilizaron encuestas y cuestionarios que ayudarían a la creación del instrumento, como el Global Physical Activity Questionnaire (GPAQv2), utilizado por Rodríguez et al. (2017) y el Cuestionario de motivaciones, actitudes y comportamientos en el ocio físico-deportivo juvenil (MACOFYD), aplicado por Ponce de León et al. (2015) y Sandoval (2017). Para la validación del instrumento, se utilizó la técnica del juicio de expertos, para la validez de contenido, seleccionando dos investigadores especialistas en el campo de la actividad física y tiempo libre; los expertos evaluaron el instrumento bajo un formato diseñado para el efecto. En cada una de las preguntas de las encuestas dieron una calificación de 1 a 5 , siendo 1 "no adecuado" y 5 "totalmente adecuado". Asimismo, los expertos realizaron observaciones y recomendaciones de cada pregunta (ítems), en términos de estructura, relevancia e importancia para la investigación.

Para la validación, se tuvo en cuenta el número de acuerdos y desacuerdos entre las valoraciones de los expertos. Se definió como acuerdo, el puntaje dado por los 2 evaluadores, que estuvieran dentro del mismo rango valorativo; así los acuerdos correspondieron a puntuaciones entre 1-4, como puntuaciones similares en el concepto o puntuaciones similares entre los valores asignados de 1 a 5 . Asimismo, se consideraron "desacuerdos", si ninguna de las puntaciones de los dos expertos coincidía. De acuerdo con lo anterior, para la estimación de la validez de los contenidos de los ítems, se obtuvo el índice de Haynes (Sabogal et al. 2011), que analiza la concordancia de jueces, obteniendo el porcentaje de acuerdo entre evaluadores, considerando una mayor proporción (de un rango entre 0,0 y 1,0), como puntaje aprobatorio de aceptación del concepto expresado por los expertos. Este índice, se calcula mediante división del número de acuerdos entre la suma de acuerdos y desacuerdos de los jueces (proporción). Así, entonces, del número total de ítems (17), los expertos presentaron desacuerdo en 6, que representa una proporción de 0,356 y presentaron acuerdo en 11 , con una proporción de 0,647 . El índice de Haynes obtenido fue de $0,647 \approx 0,65 / 1,0$, que se 
consideró aceptable, dando así, confianza sobre la validez de contenido del instrumento.

De la misma manera, se calculó el coeficiente de concordancia Kappa de Cohen (Suárez, 2015), como indicador del nivel de concordancia de los conceptos evaluativos de los dos jueces expertos. El Coeficiente kappa de Cohen es una medida estadística, que controla la aleatoriedad en la estimación de la concordancia entre evaluadores independientes (acuerdos/desacuerdos) (Campos \& Herazo, 2010) El valor de Kappa obtenido fue de 0,62, que si bien no alcanzó a ser claramente significativo, sí mostró una clara tendencia a la significancia y como tal, se puede interpretar como indicador que tiene una concordancia moderada entre los conceptos expertos, lo cual, se consideró aceptable, para asumir que el cuestionario tiene la requerida validez de contenido.

Finalmente, a fin de determinar la fiabilidad, una vez obtenido el resultado del juicio de expertos, se realizó un pilotaje con un grupo de 20 estudiantes, con características similares a la población objeto de estudio, en términos de edad y género, con el fin de encontrar sugerencias a la hora de aplicar el cuestionario. En el pilotaje, se evidenció que el cuestionario era suficientemente claro a la hora de responder, se tomó el tiempo de ejecución del instrumentoyserecibieron las preguntasysugerencias de los encuestados. Además, se utilizó el coeficiente alpha de Cronbach, cuyo valor obtenido fue de 0,72 , que se puede interpretar como una fiabilidad moderada, respecto al grado de homogeneidad de los componentes o ítems que conforman la prueba $y$, por ende, tiene propiedades, que hacen que la replicabilidad de la valoración con esta prueba en condiciones similares sea moderadamente confiable

Procedimiento. La aplicación del instrumento, se realizó con previa autorización de los rectores de los colegios y de profesores en los espacios de clases. Los estudiantes presentaron el asentimiento y consentimiento informado firmado por ellos y sus respectivos tutores legales, para la aceptación en la participación de la investigación. El diligenciamiento del cuestionario estuvo a cargo de los adolescentes, bajo la dirección del investigador principal del estudio. Esta aplicación, se realizó en un lapso de dos semanas con la anuencia previa de las directivas escolares y la colaboración de los estudiantes, para no interferir en sus actividades académicas.
Análisis de los datos. Los datos fueron procesados en el programa de análisis estadístico IBM SPSS StatisticTM versión 25.0, con previa depuración y crítica de datos para detección de inconsistencias y posibles errores en la información o en su digitación.

Se realizó un análisis descriptivo para las variables del estudio, según los ítems del cuestionario, construyendo tablas elaboradas con las frecuencias y los estadísticos de tendencia central y dispersión, según el nivel de medición de las variables, de tal manera, que para las variables cuantitativas (edad, tiempo de práctica, entre otras), se calculó la media, la mediana y la desviación estándar, mientras que para las variables cualitativas (vg. género, grado de escolaridad, actividades deportivas, expectativas, entre otras), se obtuvo la distribución porcentual de frecuencias en presentación tipo tabla.

También, se realizó un análisis correlacional, con base en el coeficiente de correlación productomomento de Pearson (Wayne, 2002), previa verificación de los supuestos de medición intervalar, tamaño muestral y normalidad, para identificar las relaciones significativas entre las variables numéricas contempladas en la encuesta, en particular las referidas a la frecuencia de prácticas deportiva, con ello se construyó la respectiva matriz de intercorrelaciones.

\section{RESULTADOS}

Los resultados de la investigación han sido estructurados en tres apartados; en el primero, se identificaron las características socio-demográficas de los adolescentes; en el segundo, se determinaron las actividades de tiempo libre y actividad física preferentes por los adolescentes; finalmente, en el tercer apartado, se identificaron las expectativas que tienen los adolescentes, frente a los programas de actividad física en el municipio.

\section{Características Socio-demográficas de los Adolescentes.}

Los resultados para la caracterización sociodemográfica, se observan en resumen en la tabla 1. Con relación al género, el total de adolescentes encuestados, el $53,4 \%$ corresponde al género masculino y el $46,6 \%$, al femenino. En cuanto a la zona de residencia, se puede observar una distribución similar por zonas (urbana y rural). Los adolescentes, se ubican en mayor proporción en instituciones 
públicas $(95,6 \%)$. En lo que respecta al estrato socio económico, el $84,8 \%$ de los estudiantes pertenecen a los estratos 1 y 2 . Los adolescentes presentan un grado de escolaridad entre octavo y once grado y porcentajes similares en cuanto a la distribución por curso

Tabla 1. Características socio-demográficas de los adolescentes del valle de san José.

\begin{tabular}{|c|c|c|c|c|c|c|c|c|c|c|c|c|}
\hline Género & \multicolumn{2}{|c|}{$\begin{array}{c}\text { Hombres } \\
159\end{array}$} & $\begin{array}{c}\% \\
53,4\end{array}$ & \multicolumn{2}{|c|}{$\begin{array}{c}\text { Mujeres } \\
139 \\
\end{array}$} & $\begin{array}{c}\% \\
46,6 \\
\end{array}$ & & & & & & \\
\hline Zona & \multicolumn{2}{|c|}{$\begin{array}{c}\text { Urbana } \\
147\end{array}$} & $\begin{array}{c}\% \\
49,3\end{array}$ & \multicolumn{2}{|c|}{$\begin{array}{c}\text { Rural } \\
151\end{array}$} & $\begin{array}{c}\% \\
50,7\end{array}$ & & & & & & \\
\hline $\begin{array}{c}\text { Tipo de } \\
\text { Institución }\end{array}$ & \multicolumn{2}{|c|}{$\begin{array}{c}\text { Pública } \\
285\end{array}$} & $\begin{array}{c}\% \\
95,6\end{array}$ & \multicolumn{2}{|c|}{$\begin{array}{c}\text { Privada } \\
13 \\
\end{array}$} & $\begin{array}{c}\% \\
4,4\end{array}$ & & & & & & \\
\hline Estrato & $\begin{array}{l}\text { E1 } \\
111\end{array}$ & $\begin{array}{c}\% \\
37,2\end{array}$ & $\begin{array}{c}E 2 \\
142\end{array}$ & $\begin{array}{c}\% \\
47,6\end{array}$ & $\begin{array}{l}\text { E3 } \\
41\end{array}$ & $\begin{array}{c}\% \\
13,7\end{array}$ & $\begin{array}{c}E 4 \\
4\end{array}$ & $\begin{array}{c}\% \\
1,30\end{array}$ & & & & \\
\hline $\begin{array}{l}\text { Grado } \\
\text { Escolar }\end{array}$ & $\begin{array}{l}8^{\circ} \\
61\end{array}$ & $\begin{array}{c}\% \\
20,5\end{array}$ & $\begin{array}{l}9^{\circ} \\
83\end{array}$ & $\begin{array}{c}\% \\
27,9\end{array}$ & $\begin{array}{l}10^{\circ} \\
85\end{array}$ & $\begin{array}{c}\% \\
28,5\end{array}$ & $\begin{array}{l}11^{\circ} \\
69\end{array}$ & $\begin{array}{c}\% \\
23,2\end{array}$ & & & & \\
\hline Edades & $\begin{array}{c}14 \\
113\end{array}$ & $\begin{array}{c}\% \\
37,9\end{array}$ & $\begin{array}{l}15 \\
78\end{array}$ & $\begin{array}{c}\% \\
26,2\end{array}$ & $\begin{array}{l}16 \\
65\end{array}$ & $\begin{array}{c}\% \\
21,8\end{array}$ & $\begin{array}{l}17 \\
30\end{array}$ & $\begin{array}{c}\% \\
10,10\end{array}$ & $\begin{array}{l}18 \\
11\end{array}$ & $\begin{array}{c}\% \\
3,7\end{array}$ & $\begin{array}{c}19 \\
1\end{array}$ & $\begin{array}{c}\% \\
0,3\end{array}$ \\
\hline
\end{tabular}

Actividades de Tiempo Libre y Actividad Física de practicar algún deporte y compartir con amigos. Las los Adolescentes. Se evidencia una alta tendencia a actividades de menor preferencia, con un interés realizar actividades sedentarias como ver televisión, menor al 17\%, corresponden a jugar video juegos, escuchar música y navegar en Internet. Mientras bailar, pintar y también, no realizar ninguna actividad tanto, las actividades de mediana preferencia son (Tabla 2). realizadas principalmente por los hombres, como

Tabla 2. a. ¿Qué actividades acostumbra a realizar en su Tiempo Libre? (fuera del colegio), b. ¿Cómo planifica su tiempo libre?

\begin{tabular}{|c|c|c|c|c|c|c|}
\hline \multicolumn{7}{|c|}{ a. actividades } \\
\hline & Frecuencia & $\%$ & Hombres & $\%$ & mujeres & $\%$ \\
\hline Ver televisión & 200 & 67,1 & 106 & 35,6 & 94 & 31,5 \\
\hline Bailar- Danzas & 22 & 7,4 & 6 & 2,0 & 16 & 5,4 \\
\hline $\begin{array}{c}\text { Practicar algún } \\
\text { deporte }\end{array}$ & 130 & 43,6 & 84 & 28,2 & 46 & 15,4 \\
\hline Navegar en internet & 154 & 51,7 & 76 & 25,5 & 78 & 26,2 \\
\hline Jugar video juegos & 50 & 16,8 & 40 & 13,4 & 10 & 3,4 \\
\hline Pintura & 19 & 6,4 & 7 & 2,3 & 12 & 4,0 \\
\hline $\begin{array}{c}\text { Compartir con } \\
\text { amigos }\end{array}$ & 124 & 41,6 & 74 & 24,8 & 50 & 16,8 \\
\hline Escuchar Música & 171 & 57,4 & 82 & 27,5 & 89 & 29,9 \\
\hline Ninguna & 5 & 1,7 & 1 & 0,3 & 4 & 1,3 \\
\hline \multicolumn{7}{|c|}{ b. planificación del tiempo libre } \\
\hline & Frecuencia & $\%$ & Hombres & $\%$ & Mujeres & $\%$ \\
\hline $\begin{array}{c}\text { Tiene planes para } \\
\text { realizar }\end{array}$ & 113 & 37,9 & 66 & 22,1 & 47 & 15,8 \\
\hline Lo deja al azar & 84 & 28,2 & 47 & 15,8 & 37 & 12,4 \\
\hline $\begin{array}{c}\text { Espera alguna } \\
\text { invitación }\end{array}$ & 52 & 17,4 & 29 & 9,7 & 23 & 7,7 \\
\hline Se queda en casa & 155 & 52,0 & 75 & 25,2 & 80 & 26,8 \\
\hline No tiene ofertas & 7 & 2,3 & 2 & 0,7 & 7 & 2,3 \\
\hline
\end{tabular}

Pregunta de selección múltiple, con múltiple respuesta. 
En cuanto a planificación del tiempo libre, se puede observar que los adolescentes prefieren, usualmente, en su tiempo libre, quedarse en casa, con un $52 \%$, seguido de un $37,9 \%$, que tiene planes para realizar, mientras que el $28,2 \%$, lo deja al azar. Un menor porcentaje espera alguna invitación o no tiene ofertas en su tiempo libre. Tanto hombres como mujeres presentan porcentaje similares en cuanto a la forma de planear lo que realizan en su tiempo libre; sin embargo, los hombres presentan una mayor tendencia a tener planes para realizar, en comparación con las mujeres (Tabla 2).
Con relación al propósito de los adolescentes al realizar AF, se observa que el factor salud, la diversión, y tener una mejor condición física son las opciones preferentes de los adolescentes. También, se evidencia que los hombres, en la opción por diversión, superan a las mujeres. Igualmente, se observa que el realizar $A F$, con el objetivo de pasatiempo, por mejorar su apariencia e integrarse con otras personas hace parte de una preferencia menor (Tabla 3).

Tabla 3. Preguntas relacionadas con la actividad física: a. ¿Con qué propósito realiza actividad física?; b. Si realiza actividad física, mencione si pertenece algún grupo deportivo o cultural; c. ¿En qué tipo de lugares prefiere pasar su TL?; $d$. ¿usted realiza algún tipo de AF durante la semana?

\begin{tabular}{|c|c|c|c|c|c|c|}
\hline \multicolumn{7}{|c|}{ a. Propósito de la actividad física } \\
\hline & Frecuencia & $\%$ & Hombres & $\%$ & Mujeres & $\%$ \\
\hline Por salud & 116 & 38,9 & 57 & 19,1 & 59 & 19,8 \\
\hline Por pasatiempo & 76 & 25,5 & 42 & 14,1 & 34 & 11,4 \\
\hline Por diversión & 111 & 37,2 & 71 & 23,8 & 40 & 13,4 \\
\hline $\begin{array}{l}\text { Por mejorar su } \\
\text { apariencia }\end{array}$ & 66 & 22,1 & 37 & 12,4 & 29 & 9,7 \\
\hline $\begin{array}{l}\text { Para tener una } \\
\text { mejor condición } \\
\text { física }\end{array}$ & 106 & 35,6 & 56 & 18,8 & 50 & 16,8 \\
\hline $\begin{array}{l}\text { Integrarse con } \\
\text { otras personas }\end{array}$ & 46 & 15,4 & 25 & 8,4 & 21 & 7,0 \\
\hline \multicolumn{7}{|c|}{ b. Grupo al que pertenece } \\
\hline & Frecuencia & $\%$ & Hombres & $\%$ & Mujeres & $\%$ \\
\hline Cultura & 7 & 2,3 & 6 & 2,0 & 1 & 0,3 \\
\hline Deportes & 41 & 13,8 & 28 & 9,4 & 13 & 4,4 \\
\hline $\begin{array}{l}\text { Caminata o } \\
\text { senderismo }\end{array}$ & 4 & 1,3 & 2 & 0,7 & 2 & 0,7 \\
\hline $\begin{array}{l}\text { Deporte y } \\
\text { cultura }\end{array}$ & 5 & 1,7 & 4 & 1,3 & 1 & 0,3 \\
\hline Ninguno & 246 & 82,6 & 124 & 41,6 & 122 & 40,9 \\
\hline \multicolumn{7}{|c|}{ c. Lugares para realizar la actividad física } \\
\hline & Frecuencia & $\%$ & Hombres & $\%$ & Mujeres & $\%$ \\
\hline Un parque & 81 & 27,2 & 42 & 14,1 & 39 & 13,1 \\
\hline $\begin{array}{l}\text { Un escenario } \\
\text { deportivo }\end{array}$ & 115 & 38,6 & 72 & 24,2 & 43 & 14,4 \\
\hline La biblioteca & 33 & 11,1 & 16 & 5,4 & 17 & 5,7 \\
\hline La casa & 144 & 48,3 & 70 & 23,5 & 74 & 24,8 \\
\hline $\begin{array}{l}\text { El medio } \\
\text { natural }\end{array}$ & 116 & 38,9 & 53 & 17,8 & 63 & 21,1 \\
\hline $\begin{array}{l}\text { Salón de video } \\
\text { juego }\end{array}$ & 31 & 10,4 & 25 & 8,4 & 6 & 2,0 \\
\hline \multicolumn{7}{|c|}{ d. actividad física semanal } \\
\hline & Frecuencia & $\%$ & Hombres & $\%$ & Mujeres & $\%$ \\
\hline $\mathrm{Si}$ & 215 & 72,1 & 124 & 41,6 & 91 & 30,5 \\
\hline No & 83 & 27,8 & 35 & 11,7 & 48 & 16,1 \\
\hline
\end{tabular}


Con relación a la participación de los estudiantes en grupos deportivos o culturales. El $82,6 \%$ de los adolescentes reportaron no pertenecer a ningún grupo de este tipo de actividades en su tiempo libre; tan solo un $13,8 \%$ hacen parte de grupos de deportes (Tabla 3).

Para los lugares que prefieren los adolescentes para pasar su TL. Se evidencia que al $48,3 \%$ de los adolescentes prefieren pasar su TL en casa; asimismo, los adolescentes eligen estar en un medio ambiente natural y escenarios deportivos, con un $38,8 \%$ y $38,6 \%$, respectivamente. Con respecto al género, se evidencia que los hombres, en un porcentaje mayor, prefieren un escenario deportivo, en comparación con las mujeres. Escenarios, como un parque, la biblioteca y el salón de video juegos son las opciones menos preferidas por los adolescentes, para asistir en su tiempo libre (Tabla 3).

Con relación a la práctica de actividad física durante la semana, se evidencia que un alto porcentaje, sí realizan algún tipo de AF. Además, se puede observar que los hombres realizan mayor AF que las mujeres (Tabla 3)

Los adolescentes que no realizan AF durante la semana reportaron, como la principal razón, a que no existen programas constantes; el $8,1 \%$ manifiestan que no existen escenarios idóneos para estas prácticas. También, se puede observar que el $6,7 \%$ no lo hace, porque le da pereza y el 6,4 reporta que no le alcanza el tiempo. Tan solo el 3\% de los adolescentes reconoce la existencia de programas, pero ninguno de estos le llama a la atención (Tabla 4).

Tabla 4. ¿Cuál cree usted que es la razón de no practicar AF o deporte?

\begin{tabular}{|l|c|c|c|c|c|c|}
\hline & Frecuencia & \% & Hombres & \% & Mujeres & $\%$ \\
\hline $\begin{array}{l}\text { No le alcanza el } \\
\text { tiempo }\end{array}$ & 19 & 6,4 & 8 & 2,7 & 11 & 3,7 \\
\hline $\begin{array}{l}\text { No existen } \\
\text { programas } \\
\text { constantes: }\end{array}$ & 38 & 12,8 & 15 & 5,0 & 23 & 7,7 \\
\hline $\begin{array}{l}\text { No existen } \\
\text { escenarios idóneos } \\
\text { para estas } \\
\text { prácticas: }\end{array}$ & 24 & 8,1 & 11 & 3,7 & 13 & 4,4 \\
\hline Le da pereza: & 20 & 6,7 & 7 & 2,3 & 13 & 4,4 \\
\hline $\begin{array}{l}\text { Si existen } \\
\text { programas en el } \\
\text { municipio, pero } \\
\text { ninguno, le llama la } \\
\text { atención: }\end{array}$ & 9 & 3,0 & 3 & 1,0 & 6 & 2,0 \\
\hline
\end{tabular}

Pregunta de selección múltiple, con múltiple respuesta.

Con relación a los minutos que dedica la población bajo estudio a la actividad física o deportiva. Se observa que existe una tendencia a dedicar minutos de su tiempo al día a realizar estas actividades, tal como señala el 78,9\% de los adolescentes consultados. La cantidad de minutos dedicados, se encuentra en un rango desde 10 a 240 minutos. El 41,4\% de los estudiantes dedican menos de 60 minutos de su tiempo a la práctica de actividad física o deportiva. El 17,1\% de los adolescentes asignan 60 minutos, siendo los hombres los que tienen una mayor frecuencia. Los adolescentes que dedican más de 60 minutos representan el 20,3\% (Tabla 5). Finalmente, se encontró que el $78,9 \%$ de los adolescentes, quienes practican deportes o realizan actividad física, dedican, en promedio, $64,1 \pm 49,7$ minutos/día. 
Tabla 5. a. ¿Cuántos minutos al día dedica a la práctica deportiva o AF?; b. ¿Cuántas veces a la semana realiza AF o deporte?

\begin{tabular}{|c|c|c|c|c|c|c|c|}
\hline \multicolumn{8}{|c|}{ a. Minutos dedicados a la práctica deportiva o actividad física } \\
\hline & Frecuencia & $\%$ & Minutos & $\mathbf{H}$ & $\%$ & M & $\%$ \\
\hline Si dedica tiempo & 235 & 78,9 & & 140 & 47,0 & 95 & 31,9 \\
\hline No dedica tiempo & 62 & 20,8 & & 18 & 6.0 & 44 & 14.8 \\
\hline \multirow{18}{*}{ Minutos al día } & & & 10 & 2 & 0,7 & 5 & 1,7 \\
\hline & & & 15 & 2 & 0,7 & 5 & 1,7 \\
\hline & & & 20 & 13 & 4,4 & 6 & 2,0 \\
\hline & & & 30 & 34 & 11,4 & 32 & 10,7 \\
\hline & & & 35 & 1 & 0,3 & 3 & 1,0 \\
\hline & & & 40 & 7 & 2,3 & 2 & 0,7 \\
\hline & & & 45 & 2 & 0,7 & 2 & 0,7 \\
\hline & & & 50 & 5 & 1,7 & 2 & 0,7 \\
\hline & & & 60 & 34 & 11,4 & 17 & 5,7 \\
\hline & & & 80 & 1 & 0,3 & 0 & 0,0 \\
\hline & & & 90 & 4 & 1,3 & 2 & 0,7 \\
\hline & & & 100 & 2 & 0,7 & 0 & 0,0 \\
\hline & & & 120 & 20 & 6,7 & 9 & 3,0 \\
\hline & & & 130 & 1 & 0,3 & 0 & 0,0 \\
\hline & & & 150 & 0 & 0,0 & 1 & 0,3 \\
\hline & & & 180 & 11 & 3,7 & 8 & 2,7 \\
\hline & & & 200 & 0 & 0,0 & 1 & 0,3 \\
\hline & & & 240 & 1 & 0.3 & 0 & 0,0 \\
\hline Total & & & & 140 & 47,0 & 95 & 31,9 \\
\hline \multicolumn{8}{|c|}{$\begin{array}{l}\text { b. veces a la semana que dedicada a la práctica deportiva o actividad } \\
\text { física }\end{array}$} \\
\hline & Frecuencia & $\%$ & veces & $\mathbf{H}$ & $\%$ & M & $\%$ \\
\hline $\begin{array}{l}\text { Si realizan } \\
\text { práctica deportiva } \\
\text { o AF: }\end{array}$ & 244 & 81,9 & & 143 & 48,0 & 101 & 33,9 \\
\hline $\begin{array}{l}\text { No realizan } \\
\text { práctica deportiva } \\
\text { o AF: }\end{array}$ & 53 & 17,9 & & 16 & 5,4 & 37 & 12,4 \\
\hline \multirow[t]{7}{*}{$\begin{array}{l}\text { Veces por } \\
\text { semana: }\end{array}$} & & & 1 & 24 & 8,1 & 18 & 6,0 \\
\hline & & & 2 & 48 & 16,1 & 31 & 10,4 \\
\hline & & & 3 & 26 & 8,7 & 28 & 9,4 \\
\hline & & & 4 & 17 & 5,7 & 9 & 3,0 \\
\hline & & & 5 & 14 & 4,7 & 7 & 2,3 \\
\hline & & & 6 & 5 & 1,7 & 5 & 1,7 \\
\hline & & & 7 & 9 & 3,0 & 3 & 1,0 \\
\hline Total & & & & 143 & 48,0 & 101 & 33,9 \\
\hline
\end{tabular}

En cuanto al promedio, el 81,9\% de los jóvenes que son quienes realizan deporte o actividad física, lo hacen $2,9 \approx$ $3,0 \pm 1,6$ días durante cada semana, encontrándose desde el adolescente, que lo hace una vez a la semana, hasta quienes diariamente desarrollan estas prácticas (Tabla 5).
Con relación a la frecuencia semanal de PD y AF de los adolescentes. Se encontró un alto porcentaje de participación semanal. Los hombres reportaron una mayor frecuencia de participación que las mujeres (Tabla 5). 
Expectativas de los adolescentes frente a los programas de AF del municipio. Pasando ahora a la descripción de las expectativas respecto a los programas municipales de AF, en la tabla 6 , se encuentran las actividades que les gustaría realizar en el TL a los adolescentes: Se encontró una alta tendencia a la práctica deportiva, siendo los deportes de conjunto, como: 1) el fútbol, el microfútbol; 2) el baloncesto y 3 ) el voleibol, los de mayor preferencia. En cuanto al género, el $42,6 \%$ de los hombres le gustaría practicar algún deporte, en contraste a las mujeres, con un $34,6 \%$.

Tabla 6. a. ¿En cuál de las siguientes actividades le gustaría ocupar su TL?; b. ¿Con quién le gustaría realizar AF?

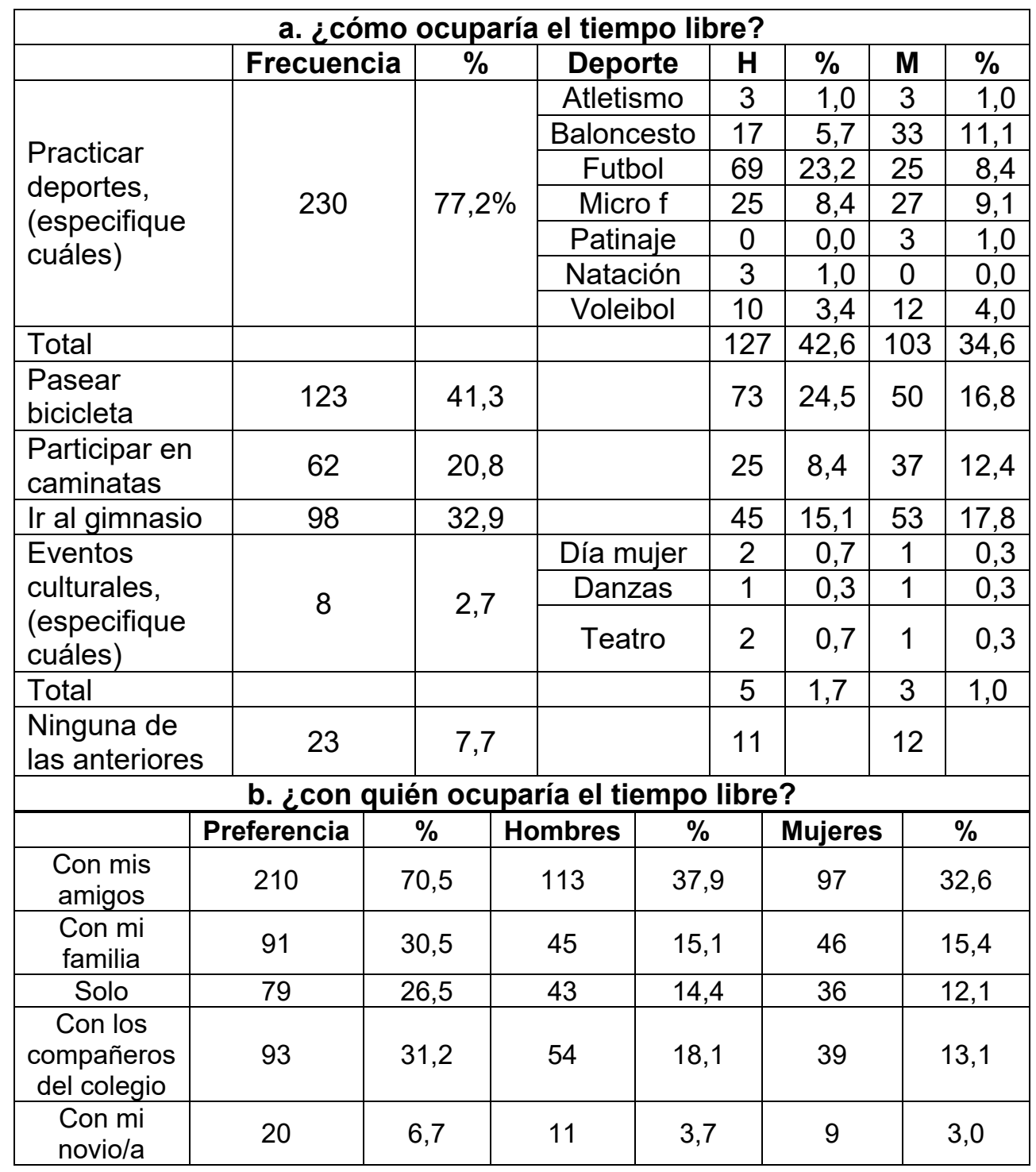

Pregunta de selección múltiple, con múltiple respuesta.

Con relación a las necesidades de compañía de los adolescentes, se encontró que a los adolescentes les gustarían practicar AF con sus amigos, con un $70,5 \%$, seguida de sus compañeros de colegio, con algún familiar y solo. En último lugar, se encuentra practicar AF con su novio/a (Tabla 6).
Con relación al conocimiento que tienen los adolescentes acerca de la existencia de programas de actividad física en el municipio donde pudieran participar en su horario extra escolar. Los resultados muestran que el $43 \%$ de los estudiantes desconocen la presencia de estos programas en el Valle de San José y el 23,1\% señaló que no hay estos programas en el municipio. En contraste, el 33,9\% restante afirman la existencia de los mismos (Tabla 7) 
Tabla 7. a. ¿Existen programas de AF en su municipio en horario extraescolar?; b. ¿Si existieran ofertas deportivas o AF con qué frecuencia asistiría por semana?; c. ¿En qué programas le gustaría ocupar su tiempo libre?; d. ¿En qué época del año le gustarían que ofrecieran los programas?

\begin{tabular}{|c|c|c|c|c|c|c|c|}
\hline \multicolumn{8}{|c|}{ a. conocimiento oferta de programas de actividad física } \\
\hline & Frecuencia & $\%$ & Hombres & $\%$ & \multicolumn{2}{|c|}{ Mujeres } & $\%$ \\
\hline $\mathrm{Si}$ & 101 & 33,9 & 58 & 19,5 & \multicolumn{2}{|c|}{43} & 14,4 \\
\hline No & 69 & 23,1 & 36 & 12,1 & \multicolumn{2}{|c|}{33} & 11,1 \\
\hline $\begin{array}{l}\text { No me he } \\
\text { enterado }\end{array}$ & 128 & 43,0 & 64 & 21,5 & \multicolumn{2}{|c|}{64} & 21,5 \\
\hline \multicolumn{8}{|c|}{ b. frecuencia con la que asistiría a realizar actividad física } \\
\hline & \begin{tabular}{l|l} 
Frecuencia \\
\end{tabular} & $\%$ & \begin{tabular}{l|l|} 
Veces \\
\end{tabular} & $\mathbf{H}$ & $\%$ & $\mathbf{M}$ & $\%$ \\
\hline Si asistirían & 253 & 84,9 & & 136 & 45,6 & 117 & 39,3 \\
\hline No asistirían & 44 & 15,1 & & 22 & 7,4 & 22 & 7,4 \\
\hline \multirow{7}{*}{ Veces por semana } & & & 1 & 12 & 4,0 & 16 & 5,4 \\
\hline & & & 2 & 52 & 17,4 & 39 & 13,1 \\
\hline & & & 3 & 37 & 12,4 & 28 & 9,4 \\
\hline & & & 4 & 13 & 4,4 & 7 & 2,3 \\
\hline & & & 5 & 16 & 5,4 & 16 & 5,4 \\
\hline & & & 6 & 1 & 0,3 & 3 & 1,0 \\
\hline & & & 7 & 5 & 1,7 & 8 & 2,7 \\
\hline Total & & & & 136 & 45,6 & 117 & 39,3 \\
\hline \multicolumn{8}{|c|}{ c. cómo ocuparía su tiempo libre } \\
\hline & Frecuencia & $\%$ & Hombres & $\%$ & $\mathrm{Mu}$ & jeres & $\%$ \\
\hline $\begin{array}{l}\text { Actividades } \\
\text { deportivas }\end{array}$ & 204 & 68,5 & 120 & 40,3 & & 84 & 28,2 \\
\hline A. físicas & 129 & 43,3 & 64 & 21,5 & & 65 & 21,8 \\
\hline A. recreativas & 84 & 28,2 & 36 & 12,1 & & 48 & 16,1 \\
\hline A. Culturales & 65 & 21,8 & 25 & 8,4 & & 40 & 13,4 \\
\hline Ninguno & \begin{tabular}{l|l}
8 & \\
\end{tabular} & 2,7 & 4 & 1,3 & & 4 & 1,3 \\
\hline \multicolumn{8}{|c|}{ d. cuando realizaría actividad física } \\
\hline & Frecuencia & $\%$ & Hombres & $\%$ & \multicolumn{2}{|c|}{ Mujeres } & $\%$ \\
\hline $\begin{array}{l}\text { En vacaciones de } \\
\text { mitad de año }\end{array}$ & 128 & 43,0 & 65 & 21,8 & \multicolumn{2}{|r|}{63} & 21,1 \\
\hline $\begin{array}{l}\text { En vacaciones de } \\
\text { octubre }\end{array}$ & 30 & 10,1 & 17 & 5,7 & \multicolumn{2}{|c|}{13} & 4,4 \\
\hline $\begin{array}{l}\text { En vacaciones de } \\
\text { fin de año }\end{array}$ & 57 & 19,1 & 21 & 7,0 & \multicolumn{2}{|c|}{36} & 12,1 \\
\hline $\begin{array}{l}\text { En la época } \\
\text { académica }\end{array}$ & 33 & 11,1 & 22 & 7,4 & \multicolumn{2}{|r|}{11} & 3,7 \\
\hline En todo el año & 164 & 55,0 & 83 & 27,9 & \multicolumn{2}{|r|}{81} & 27,2 \\
\hline
\end{tabular}


Con relación con la frecuencia en términos de veces por semana, con la que asistirían a realizar actividades deportivas o $A F$, en caso de que existiera su oferta en el municipio. El 84,9\% de los adolescentes señaló su posible participación en determinado número de veces por semana (Tabla 7). Se puede indicar, que los estudiantes que expresan que sí asistirían a la oferta deportiva es del $84,9 \%$. En promedio, lo harían $3,0 \pm 1,5$ veces/semana a las actividades deportivas y el ejercicio físico.

Respecto a los programas que les gustarían a los adolescentes, los programas deportivos son de mayor preferencia, con un $68,5 \%$. Lo anterior fue informado mayoritariamente por los hombres, mientras que las mujeres, un $28,2 \%$. Programas de actividad física, con el $43,3 \%$, se presenta como la segunda preferencia. Por su parte, los adolescentes que manifiestan no gustarle ningún tipo de programa, representan una minoría, con el 2,7\% (Tabla 7).

En cuanto a la época del año preferente para la oferta de estos programas. Los estudiantes señalaron, en mayor porcentaje, les gustaría que los programas se ofrecieran durante todo el año. Un 43\% prefiere que se realicen en vacaciones de mitad de año y en menores porcentajes, se encuentran las vacaciones de fin de año, las de octubre y en época académica (Tabla 7).
El análisis de las intercorrelaciones, se realizó entre las variables numéricas, que fueron contempladas en el cuestionario de caracterización de las actividades de tiempo libre y las expectativas frente a los programas de actividad física de los adolescentes del municipio Valle de San José, como fueron la frecuencia (número de veces/ semana) de práctica deportiva que los jóvenes realizan semanalmente de alguna práctica deportiva o de AF; el tiempo que se dedica y la frecuencia que realizarían, si hubiese una oferta de programas con mayor variedad. Para ello, se consideraron solamente a los participantes de la población, que respondieron afirmativamente a las preguntas respectivas del tiempo o las frecuencias de prácticas deportivas.

De la matriz correlacional de la tabla 8, se puede afirmar que, basado en la aplicación del coeficiente de correlación de Pearson, se encontró correlación significativa inversa, aun cuando baja, entre la edad de los estudiantes y el tiempo día (minutos/día), que se dedica a la práctica deportiva y la $A F$, de manera que, con $r=-0.169$, se puede afirmar que, en una porción de los estudiantes adolescentes, se da que los de mayor edad dedican un menor tiempo a la realización de deportes, mientras que los de menor edad, dedican mayor cantidad de tiempo al día a la actividad física.

Tabla 8. Matriz correlacional.

\begin{tabular}{|c|c|c|c|c|c|}
\hline \multicolumn{6}{|c|}{ Correlaciones } \\
\hline & & Edad & $\begin{array}{c}\text { Minutos/ } \\
\text { día } \\
\text { PD AF }\end{array}$ & $\begin{array}{l}\text { \# Veces/sem } \\
\text { PD AF }\end{array}$ & $\begin{array}{c}\# \\
\text { Veces/sem } \\
\text { Asistiría } \\
\text { PD AF } \\
\end{array}$ \\
\hline \multirow{3}{*}{ Edad } & $\begin{array}{c}\text { rde } \\
\text { Pearson }\end{array}$ & 1 &,$- 169^{\star *}$ & ,094 &,- 029 \\
\hline & Sig. & & ,009 & , 145 & ,626 \\
\hline & $\mathrm{N}$ & 298 & 235 & 244 & 285 \\
\hline \multirow{3}{*}{$\begin{array}{l}\text { Minutos/día } \\
\text { PD AF }\end{array}$} & $\begin{array}{c}r \text { de } \\
\text { Pearson }\end{array}$ & $\begin{array}{c}- \\
169 * \\
*\end{array}$ & 1 &, $187^{* *}$ & ,122 \\
\hline & Sig. & ,009 & & ,004 & ,061 \\
\hline & $\mathrm{N}$ & 235 & 235 & 234 & 235 \\
\hline \multirow{3}{*}{$\begin{array}{l}\text { \# Veces/sem } \\
\text { PD AF }\end{array}$} & $\begin{array}{c}r \text { de } \\
\text { Pearson }\end{array}$ & ,094 &, $187^{* *}$ & 1 &, $196^{* *}$ \\
\hline & Sig. & , 145 & ,004 & & ,002 \\
\hline & $\mathrm{N}$ & 244 & 234 & 244 & 242 \\
\hline \multirow{3}{*}{$\begin{array}{l}\text { \# Veces/sem } \\
\text { Asistiría PD } \\
\text { AF }\end{array}$} & $\begin{array}{c}\text { rde } \\
\text { Pearson }\end{array}$ &,- 029 & ,122 &, $196 * *$ & 1 \\
\hline & Sig. & ,626 & ,061 & ,002 & \\
\hline & $\mathrm{N}$ & 285 & 235 & 242 & 285 \\
\hline
\end{tabular}

**. La correlación es significativa en el nivel 0,01 (bilateral). 
También, se encontró correlación significativa directa $(p=0,004)$ con $r=0,187$, entre la cantidad de minutos que se emplean en las prácticas de AF y deporte y el número de sesiones a la semana que los jóvenes informan dedicar al deporte y AF que, aunque es evidente, permite afirmar que, quienes con más frecuencia asisten a sesiones de práctica mayor, dedican un mayor tiempo a la práctica de actividades físicas y deportivas. Finalmente, el análisis correlacional dejó ver que el tiempo de realización diaria actual de práctica deportiva se relaciona significativamente, con la expectativa de asistir mayor número de veces a los programas que se oferten en el área de la actividad física, dado que estas variables correlacionaron directa y significativamente $(p=0,002)$, con un $r=0,196$.

\section{DISCUSIÓN}

Con relación a las actividades que realizan en el tiempo libre los adolescentes del municipio del Valle de San José, se encontró que actividades, como ver Tv, navegar por Internet y escuchar música, presentan una frecuencia mayor del $50 \%$. Estos resultados coinciden con los hallazgos reportados por Huertas et al. (2008), quienes señalan que las actividades de ocio de mayor preferencia en los adolescentes y jóvenes universitarios están referidas al llamado ocio pasivo o audiovisual, que involucran este tipo de actividades.

Respecto a la planificación de las actividades por parte de los adolescentes, se encontró que en su tiempo libre prefiere quedarse en casa, mientras un menor porcentaje, planifica lo que va a realizar o lo deja al azar. Estos resultados están relacionados con las preferencias de ocio pasivo registradas, debido a que este tipo de actividades son realizadas, generalmente, en sus residencias. En contraste, al estudio realizado por Sandoval (2017) muestra una mayor frecuencia en estudiantes que dejan al azar las actividades a realizar (37\%). Según el autor, esto se encuentra posiblemente asociado, con querer evitar la monotonía.

Al $77,2 \%$, de los adolescentes, en su tiempo libre, les gustaría realizar deportes de conjunto, siendo baloncesto, fútbol, micro fútbol y voleibol, los más preferidos, con un $73,3 \%$, frente a los deportes individuales (patinaje, atletismo y natación), con un 3,9\%. Una situación similar ocurre en otras investigaciones: Codina et al. (2016) encontraron que el $44,7 \%$ de los adolescentes prefieren los deportes de conjunto y el $16,5 \%$, los deportes individuales. Igual tendencia muestra la investigación de Vergara et al. (2015), hallando que el $51,5 \%$ de los adolescentes prefieren los deportes de conjunto; no obstante, en el presente estudio, la incorporación de los adolescentes en los diferentes grupos culturales y deportivos es bastante baja; de hecho, el $82,6 \%$ no pertenece a ninguna de estas formas de participación social.

En cuanto al propósito que tiene para realizar actividad física, los adolescentes encuestados señalan al factor salud como el más importante, con porcentajes similares al factor diversión y al tener una mejor apariencia física. Esto coincide con lo hallado por Rodríguez etal. (2017), en su estudio sobre los motivos para la práctica de actividad física durante el tiempo libre, en el que los encuestados destacan al factor salud, como el más importante, a la hora de realizar una práctica de actividad física en su TL.

Lema et al. (2009) también expone que las principales razones que aluden las personas para realizar actividad física están la diversión, mejorar la salud, mejorar la imagen y encontrarse con los amigos. En contraste, para no realizar alguna práctica deportiva o de actividad física, la principal razón, según los adolescentes del Valle de San José, es atribuida a que no existen programas constantes, además de la falta de escenarios y otras razones, como la pereza y la falta de tiempo. Estas últimas razones, también fueron reportadas por Valera et al. (2011), en el estudio sobre actividad física y sedentarismo en jóvenes universitarios de Colombia; los adolescentes señalaron, como motivo principal para no hacer o dejar de hacer actividad física, a la pereza (61,5\%). Como expresan los autores, este tipo de factores podría estar relacionado con una cultura juvenil que puede tender al sedentarismo, en la que los jóvenes poco se cuidan, a pesar de privilegiar el cuerpo y centrarse en él.

En el presente estudio, los resultados mostraron una alta participación de los adolescentes en prácticas de actividad física. El $72,1 \%$ reportó que realizan actividad física durante la semana. Lo anterior guarda relación con lo informado por otros estudios realizados en este mismo tipo de población; por ejemplo, Ponce de León et al. (2015) encontraron una alta participación de su muestra en actividades físicas $(63,9 \%)$. Rubio \& Varela (2016), también registraron que el $75 \%$ de los jóvenes encuestados realizan actividad física. En relación al género, según los datos obtenidos, los hombres son más activos físicamente que las mujeres. Estas diferencias también fueron halladas por Rodríguez etal. (2017), Pérez et al. (2014), Cossio et al. (2015), Vergara et al. (2015), Valdés \& Yanci (2016) y Ponce de León et al. (2015). 
Pese a la alta participación en prácticas de actividad física, se encontró que tan solo el $37,4 \%$ de los adolescentes dedican, como mínimo, 60 minutos diarios en actividades físicas. Además, solo el 41,3\% incorporan, como mínimo, 3 veces por semana alguna práctica deportiva o actividad física. Lo anterior, evidencia que un alto porcentaje de los encuestados no cumplen con lo recomendado por la OMS, que indica que los adolescentes deben invertir, mínimo, 60 minutos diarios, incorporados 3 veces por semana (Organización Mundial de la Salud, 2020).

Otro de los aspectos de interés concerniente con la realización de actividad física es que un gran porcentaje de adolescentes busca contar con la compañía de otras personas, al realizar este tipo de actividades. Estos resultados anteriores, no coinciden con los hallazgos reportados por Carmona (2015), quién encontró un porcentaje más bajo con relación a este componente social, debido a que el $52 \%$ de los encuestados nunca o algunas veces comparten con su familia o amigos. Por su parte, a los adolescentes del Valle de San José, les gustaría practicar AF con sus amigos y con su familia, con porcentajes de 30,5\% y 70,5\%, respectivamente. Esta notable diferencia entre la preferencia de compañía de amigos y familia, podría evidenciar débiles conexiones entre la población adolescente y las redes de apoyo familiares. Estas redes, según Páez (2012), inciden en la adopción de estilos de vida en adolescentes y jóvenes, por lo cual, es necesario trabajar en su fortalecimiento.

Por otra parte, en cuanto a la percepción que tienen los estudiantes acerca de los programas de actividad física, tan solo el 33,9\% de los adolescentes señalan que existen este tipo de programas; los demás, reportan que no existen o que no se han enterado. Esta percepción, se podría deber a una falta de conocimiento de dichos programas, posiblemente, por una falla en la promoción de los mismas. Lo anterior, sugiere la necesidad de incluir mayor información de los programas que se ofrecen y la manera en que adolescentes pueden acceder a ellos.

Los adolescentes también señalaron, en su mayoría, que de existir este tipo de ofertas deportivas y de actividad física, asistirían. De hecho, un $84,9 \%$ reportó que lo harían y la frecuencia semanal preferente sería hasta tres veces, de existir este tipo de ofertas deportivas y de actividad física. De hecho, un 84,9\% reportó que lo harían y la frecuencia semanal, en promedio, sería de $3,0 \pm 1,5$ veces/semana. Los programas de preferencia por los adolescentes son los deportivos seguidos de los de actividad física en general. Además, señalaron, en mayor porcentaje, que les gustaría que los programas se ofrecieran durante todo el año. Estos datos revelan el interés que presentan los adolescentes del Valle de San José, por participar en programas de actividad física y de prácticas deportivas y el desconocimiento que tienen sobre la existencia de los mismos. Este tipo de programas para fomentar la actividad física, según Muros et al. (2016), son necesarios, para crear hábitos saludables en edades tempranas, evitando el aumento del sedentarismo.

Existe una relación significativa inversa de $r=0,169$ entre la edad de los estudiantes y el tiempo que dedican al día a la práctica de AF o deportiva, donde se da un resultado inversamente proporcional, debido a que, entre más edad de los adolescentes, menor es la práctica deportiva, por lo tanto, en menor edad mayor es la frecuencia en estas prácticas. Esto coincide con Meneses \& Ruíz (2017), quienes reportaron que, a medida que pasan los años, se evidencia el abandono de la práctica de actividad física y deportiva en el tiempo libre; no obstante, estos resultados no coinciden con los hallazgos de Pratap \& Misra (2015), quienes manifiestan que los adolescentes de edades tempranas (12-14 años), dedican más tiempo a realizar actividades sedentarias, a diferencia de los adolescentes de edades tardías (17-18 años), los cuales, son más activos.

\section{CONCLUSIONES}

La investigación realizada permitió identificar las características sociodemográficas de los adolescentes del municipio del Valle de San José, como género, zona de residencia, tipo de institución a la que pertenecen, estrato socio económico, grado de escolaridad y sus edades. De acuerdo con los resultados obtenidos es importante que el municipio oferte programas de actividad física, disponibles para toda la población adolescente, considerando que un alto porcentaje pertenece a los estratos socioeconómicos 1 y 2 , para los cuales, no es tan fácil acceder a ofertas realizadas por entidades privadas. Además, se tendrá que analizar, con mayor detalle, el papel que juegan otros factores, como la zona de residencia, debido a que el $50 \%$ de los adolescentes viven en la zona rural y esto puede representar un impedimento para participar de los programas que se ofrecen en el municipio.

Los resultados de este estudio aportan información sobre las actividades que realizan los adolescentes en su tiempo libre y la práctica de actividad física. Se evidenció 
que un alto porcentaje de adolescentes realizan AF; sin embargo, el tiempo destinado y la frecuencia de realización para este tipo de actividades son inferiores a las recomendaciones señaladas por los diferentes organismos internacionales para obtener objetivos saludables. Lo anterior, se encuentra relacionado con la preferencia de otro tipo de actividades de ocio pasivo, que se están dando en nuestra sociedad. Sería necesario aumentar el interés de los adolescentes hacia una práctica más frecuente de AF, sobre todo en el caso de las mujeres, quienes presentan menor participación, en este tipo de actividades.

Los adolescentes mostraron un alto deseo y significativo $(p=0,002)$, con un $r=0,196$, con relación al tiempo que dedican a realizar actividad física y las expectativas de asistencia en futuros programas de AF. Los deportes de conjuntoy el ciclismo, se presentaron comolas actividades físicas de mayor preferencia, las cuales, realizarían acompañados por sus amigos. A los adolescentes les gustaría que estos programas fueran ofertados continuamente durante todo el año y manifiestan una alta disponibilidad de asistir frecuentemente a las sesiones semanales.

Los hallazgos obtenidos en esta investigación pueden servir de utilidad para las instituciones del municipio, en la creación y refuerzo de los programas de intervención, que estarían basados en las necesidades y preferencias reales de los adolescentes, dirigidos al fomento de la práctica de AF y la obtención de beneficios para la salud en esta población.

Limitaciones del estudio. Como limitaciones de este estudio, cabe señalar que, por temas de disponibilidad, no se incluyeron a otros actores que intervienen en el tema de investigación, como los docentes de educación física, coordinadores culturales y las entidades responsables de la creación, ejecución y seguimiento de los programas de actividad física.

Sobre la base de esto, se considera importante incluir futuras investigaciones que superen estas limitaciones metodológicas. Además, dichos estudios, se podrían dirigir a la comprensión de los factores, como la continuidad de la práctica de actividad física por parte de los adolescentes y cualificar la oferta de los programas de promoción de estas prácticas. Además, se recomienda a las entidades encargadas de la creación y la ejecución de programas para el aprovechamiento del tiempo libre, realizar la promoción, el monitoreo y la evaluación de estos programas, con el fin de mejorar y aumentar la participación de los adolescentes y el alcance de estas iniciativas.

De acuerdo con el estudio, se recomienda tener en cuenta las expectativas que tienen los adolescentes frente a los programas en términos de: 1) Frecuencia semanal de participación; 2) Época del año preferente y 3) Los tipos de actividades que les gustaría realizar.

\section{REFERENCIAS}

1. BAENA, A.; GRANERO, A. 2015. Educación física e intención de práctica física en tiempo. Revista Electrónica de Investigación Educativa. 17(3):132-144.

2. CAMPOS, A.; HERAZO, E. 2010. Concordancia intra e inter-evaluadores. Colomb Psiquiat. 39(2):424-432. https://doi.org/10.1016/S00347450(14)60261-4

3. CARMONA, V. 2015. Aprovechamiento del tiempo libre y realización de actividad física regular en comunidad universitaria de Colombia. Prueba Piloto. Revista CES Movimiento y Salud. 3(1):16-22.

4. CODINA, N.; PESTANA, J.; CASTILLO, I.; BALAGUER, I. 2016. Ellas a estudiar y bailar, ellos a hacer deporte": Un estudio de las actividades extraescolares de los adolescentes mediante los presupuestos de tiempo. Cuadernos de Psicología del Deporte. 16(1):233-242.

5. COSSIO, M.; VIVEROS, F.; CASTILLO, M. 2015. Patrones de actividad física en adolescentes en función del sexo, edad cronológica y biológica. Nutrición clínica y Dietética hospitalaria. 35(2):41-47. https://doi.org/10.12873/352cossio

6. GALVIN, M. 2019. Actividad física y su hijo de 13- 18 años. KidsHealth. Disponible desde Internet en: https://kidshealth.org/es/parents/fitness-1318-esp.html

7. HUERTAS, C.; CARO, D.; VÁSQUEZ, A.; VÉLEZ, J.S. 2008. Consumo cultural y uso del tiempo libre en estudiantes Lasallistas. Revista Lasallista de investigación. 5(2):36-47.

8. LEMA, L.; SALAZAR, I.; VALERA, M.; TAMAYO, J.; RUBIO, A.; BOTERO, A. 2009. Comportamiento y salud de los jóvenes universitarios: satisfacción con el estilo de vida. Pensamiento psicológico. 5(12):71-87. 
9. MENESES, M.; RUÍZ, F. 2017. Estudio longitudinal de los comportamientos y el nivel de actividad físico-deportiva en el tiempo libre en estudiantes de Costa Rica, México y España. Retos: Nuevas perspectivas de educación física, deporte y recreación. 31:219-226.

https://doi.org/10.47197/retos.v0i31.53396

10. MUROS, J.; COFRE, B.; SALVADOR-PÉREZ, S.; CASTRO-SÁNCHEZ, M.; VALDIVIA-MORAL, P.; PÉREZ, A.J. 2016. Relación entre nivel de actividad física y composición corporal en escolares de Santiago (Chile). Journal of sport and health research. 8(1):65-74.

11. ORGANIZACIÓN MUNDIAL DE LA SALUD. 2020. Estrategia mundial sobre regimen alimentario, actividad fisica y salud. OMS. Disponible desde Internet en:

https://www.who.int/dietphysicalactivity/pa/es/

12. PÁEZ, M. 2012. Universidades saludables: los jóvenes y la salud. Archivos de Medicina (Col). 12(2):205-220.

13. PÉREZ, G.; LAIÑO, F.; ZELARAYAN, J.; MÁRQUEZ, S. 2014. Actividad fisica y habitos de salud en estudiantes universitarios argentinos. Nutrición hospitalaria. 30(4):896-904.

14. PONCE DE LEÓN, A.; SANZ, E.; VALDEMOROS, A. 2015. Ocio familiar y Actividad física en estudiantes de bachillerato. Pedagogía social, revista interuniversitaria. 25:51-68.

15. PRATAP, A.; MISRA, G. 2015. Pattern of leisurelifestyles among Indian school adolescents: Contextual influences and implications for emerging health concerns. Cogent Psychology. 2(1):1-11. https://doi.org/10.1080/23311908.201 5.1050779

16. RODRÍGUEZ, R.; MACÍAS, P.; GARRIDO, M.; TEJERO, C.; LÓPEZ, A. 2017. Motivos para la práctica de actividad física durante el tiempo libre y su relación con el cumplimiento de las recomendaciones. Cuaderno de la psicología del deporte. 18(1):183-194.

17. RUBIO, R.; VARELA, M. 2016. Barreras percibidas en jóvenes universitarios para realizar actividad física. Revista Cubana de salud pública. 42(1):61-69.

18. SABOGAL, L.; BARRAZA, E.; HERNÁNDEZ, A.; ZAPATA, L. 2011. Validación del cuestionario de motivación y estrategias de aprendizaje en estudiantes universitarios de una Institución pública. Psicogente. 14(35):36-50.
19. SANDOVAL, N. 2017. Diagnóstico acerca del uso del ocio y el tiempo libre entre los estudiantes de la Universidad Nacional Experimental del Táchira. Pedagogía Social. Revista Interuniversitaria. 30:169-188.

https://doi.org/10.7179/PSRI_2017.30.12

20. SUÁREZ, A. 2015. La estadística en la validación de escalas, una visión práctica para su construcción o su adaptación. Revista 13+. 2(2):46-61. https://doi.org/10.24267/23462329.111

21. VALDÉS, P.; YANCI, J. 2016. Análisis de la condición física, tipos de actividad física realizada y rendimiento académico en estudiantes de educación secundaria. Retos. 30:64-69. https://doi.org/10.47197/retos.v0i30.36862

22. VALERA, M.; DUARTE, C.; SALAZAR, I.; LEMA, L.; TAMAYO, J. 2011. Actividad física y sedentarismo en jóvenes universitarios de Colombia: prácticas, motivos y recursos para realizarlas. Colombia Médica. 42(3):269-277.

23. VERGARA, M.; ELZEL-CASTRO, L.; RAMÍREZ, R.; CASTRO, R.; CACHÓN-ZAGALAZ, J. 2015. Actividad física en estudiantes de octavo curso de educación básica de la comuna de Río Bueno (Chile). Journal of and Health Research. 7(1):43-54.

24. WAYNE, D. 2002. Bioestadística: Bases para el análisis de las ciencias de la salud (Vol. 4). Limusa Wiley (México). 915p. 